\title{
QUASI-DISJOINTNESS IN ERGODIC THEORY $\left({ }^{1}\right)$
}

\author{
BY
}

\section{KENNETH BERG}

\begin{abstract}
We define and study a relationship, quasi-disjointness, between ergodic processes. A process is a measure-preserving transformation of a measure space onto itself, and ergodicity means that the space cannot be written as a disjoint union of invariant pieces, unless one of the pieces is of zero measure. We restrict our attention to spaces of total measure one which also satisfy additional regularity properties. In particular, the associated Hilbert space of square-summable functions is separable. A simple class of examples is given by translation by a fixed element on a compact Abelian metrizable group, such processes being known as Kronecker processes. We introduce the notion of a maximal common Kronecker factor (or quotient) process for two processes. Quasi-disjointness is a notion tied to the homomorphisms from two processes into their maximal common Kronecker factor, and reduces to a previous notion, disjointness, when that factor is trivial. We show that a substantial class of processes, the Weyl processes, are quasi-disjoint from every ergodic process. As a corollary, we show that a Weyl process and an ergodic process are disjoint if and only if they have no nontrivial Kronecker factor in common, or, equivalently, if they form an ergodic product. We give an example which suggests an analogous theory could be constructed in topological dynamics.
\end{abstract}

0. Introduction. The existence of common eigenvalues other than 1 for ergodic processes implies the existence of nontrivial common factors, and so precludes disjointness. It is known [7] that for certain classes of processes, this is the sole impediment to disjointness, i.e., if the processes have no common eigenvalue other than 1 then the processes are disjoint. We employ a certain mapping from the product of two processes into the Kronecker process which has for its spectrum the eigenvalues common to the two factors to decompose the phase space of the product. The ergodic disintegration of the product measure provides for a measure with certain compatibility properties on almost every one of the pieces. Processes are quasi-disjoint if there is only one such measure on almost all of the pieces. We show that quasi-disjointness is preserved under ergodic group extension and passage to factors. We give two examples, one of which suggests the possibility of an analogous theory in topological dynamics.

Presented to the Society, January 231970 under the title Quasi-disjointness in ergodic theory, Weyl processes; received by the editors February 26, 1970.

AMS 1968 subject classifications. Primary 2670; Secondary 4730, 5482, 6005.

Key words and phrases. Ergodic process, spectral measure, Weyl process, ergodic group extension, disjoint processes, ergodic decomposition, disintegration of a measure, Kronecker process.

(1) This research was supported in part by the National Science Grant GP 9605.

Copyright (C) 1972, American Mathematical Society 
We keep a close watch on sets of measure zero throughout the paper. Although tedious, we felt this was necessary for clarity and precision. Perhaps overall it serves the cause of brevity as well. One aspect of this attitude is that we work with standard Borel spaces instead of Lebesgue spaces. The latter notion is tied to the particular measure on the space, while in our study we wish to regard the measure as a variable.

We wish to point out that our results overlap with those of Hahn and Parry mentioned above. We also wish to acknowledge useful conversations with $\mathbf{J}$. Auslander, A. Kleppner, and N. Markley.

1. Preliminary results and notation. In this section we set out our basic notation, recall some known facts, and describe our intentions. We also prove certain results which, perhaps, are part of the folklore but which we could not find in print.

A process $X$ is a quadruple $\left(\Omega_{X}, \mathscr{F}_{X}, \mu_{X}, T_{X}\right)$ where $\left(\Omega_{X}, \mathscr{F}_{X}\right)$ is a standard Borel space (i.e., $\Omega$ admits a complete separable metric such that $\mathscr{F}$ consists of the Borel $\sigma$-field; here as elsewhere we omit the subscript $X$ on $\Omega, \mathscr{F}$, etc. when no confusion can arise), $\mu_{X}$ is a probability measure on $\left(\Omega_{X}, \mathscr{F}_{X}\right)$, and $T_{X}$ is a bimeasurable bijection of $X$ onto itself satisfying $\mu(T F)=\mu(F)$ for all measurable $F$. Basic information on standard Borel spaces can be found in [11], and related facts can be found (in some form) in [14], [12], and [3]. We mention several facts which will be of particular interest to us.

(1) If a function maps one standard Borel space into another in a one-to-one and measurable fashion, then the range of the function is measurable, the restriction of the $\sigma$-field of the image space to the range makes the range into a standard Borel space, and the inverse mapping is also measurable. In particular, it suffices in the definition of a process to say $T$ is a measurable (rather than bimeasurable) bijection. See [11, Theorem 3.2].

(2) If a function maps one standard Borel space into another in a measurable fashion (not necessarily one-to-one) then the range, while not necessarily measurable, is in the universal completion of the $\sigma$-field of the range space. Probably the easiest way to see this is to use the idea of a measurable section, as given in [3], together with the aforementioned facts about one-to-one mappings (and the fact that any Borel subset of a standard Borel space is a standard Borel space with the inherited $\sigma$-field. This is Corollary 1 of Theorem 3.2 of [11]). In order to use Bourbaki, we must know the space can be given a locally compact topology compatible with the $\sigma$-field. In fact, the space is either countable or can be given the topology of the unit interval [11, p. 138]. It is also possible to deduce the fact in question by using the theory of analytic spaces.

(3) It is possible to represent (in a sense to be described) any process as a shift on an appropriate product space (but not with the product measure, in general). We will explain this for the case when $\Omega$ consists of uncountably many points. In this case there exists a sequence $F_{1}, F_{2}, \ldots$ of measurable sets such that if $t$ is any mapping of $N=\{1,2,3, \ldots\}$ into $Z_{2}=\{0,1\}$ then there is a unique $x=x_{t}$ in $\Omega$ 
such that $x$ is in $F_{i}$ if and only if $t(i)=1\left[11\right.$, p. 138]. If we set $\Omega_{1}=Z_{2}^{N}$ and $\Omega_{2}=\Omega_{1}^{Z}$ $(Z=\{0, \pm 1, \ldots\})$ then $\Omega_{X}$ can be mapped into $\Omega_{2}$ by

$$
\begin{aligned}
\sigma(x)(i)(n) & =1 & & \text { if } T^{i} x \in F_{n}, \\
& =0 & & \text { otherwise. }
\end{aligned}
$$

The mapping $\sigma$ is obviously measurable and one-to-one so the range is a Borel set (we give $Z_{2}$ the discrete topology and $\Omega_{1}$ and $\Omega_{2}$ the product topology and the $\sigma$-field for $\Omega_{2}$ is describable either as the Borel field for the compact metric topology or as the product field). We set $\mu_{2}(F)=\mu_{X}\left(\sigma^{-1}(F)\right)$ so that the range has measure one. If we now define the shift $T_{2}$ on $\Omega_{2}$ by $\left(T_{2} \omega\right)(i)=\omega(i+1)$ we see that $T_{2} \sigma(x)$ $=\sigma T_{X}(x)$ for all $x$ in $\Omega$.

The representation constructed above is an instance of isomorphism mod 0 , which we describe shortly. If $X_{1}$ and $X_{2}$ are processes and $\pi: \Omega_{1} \rightarrow \Omega_{2}$ is a bimeasurable bijection satisfying $\mu_{2}(\pi F)=\mu_{1}(F)$ and $T_{2} \pi(x)=\pi T_{1} x$ for all $F$ in $\mathscr{F}_{1}$, $x$ in $\Omega_{1}$ then we say $\pi$ is an isomorphism. In case there exists invariant null sets $N_{1} \subseteq \Omega_{1}$ and $N_{2} \subseteq \Omega_{2}(N$ is invariant means $T N=N)$ such that there is a mapping $\pi: \Omega_{1} \backslash N_{1} \rightarrow \Omega_{2} \backslash N_{2}$ with the above properties, we say $\pi$ is an isomorphism $(\bmod 0)$. Most interesting measure-theoretic properties of systems (e.g., spectral properties, entropy) are insensitive to replacement of a process by an isomorphic $(\bmod 0)$ copy. Now suppose $\pi$ is measurable, not necessarily one-to-one or onto, and satisfies $\mu_{1}\left(\pi^{-1}(F)\right)=\mu_{2}(F)$ and $T_{2} \pi(x)=\pi\left(T_{1}(x)\right)$. We then say $\pi$ is a homomorphism, $X_{2}$ is a factor of $X_{1}$, and $X_{1}$ is an extension of $X_{2}$. Note that the range of $\pi$ has measure one with respect to the completion of $\mu_{2}$. In case the domain of definition of $\pi$ is only a set of measure one, we say $\pi$ is a homomorphism $(\bmod 0)$. It is not difficult to see that if $\pi: X_{1} \rightarrow X_{2}$ is a homomorphism (mod 0$)$ then, by deleting invariant null sets from $\Omega_{1}$ and $\Omega_{2}$, we have processes $X_{1}^{\prime}$ and $X_{2}^{\prime}$ isomorphic $(\bmod 0)$ to $X_{1}$ and $X_{2}$ such that the restriction of $\pi$ to $X_{1}^{\prime}$ is a homomorphism. Since any invariant set in $X_{2}$ which is of measure zero for the completion of $\mu_{2}$ is contained in an $\mathscr{F}_{2}$-measurable null invariant set, we can in fact choose $X_{1}^{\prime}$ and $X_{2}^{\prime}$ so that the restriction of $\pi$ to $X_{1}^{\prime}$ is surjective on $X_{2}^{\prime}$. Also we observe that if $\pi$ is a homomorphism $(\bmod 0)$ of $X_{1}$ to $X_{2}$ and if $X_{i}^{\prime}$ is isomorphic $(\bmod 0)$ to $X_{i}$ $(i=1,2)$ then there is a naturally defined homomorphism of $X_{1}^{\prime}$ to $X_{2}^{\prime}$. In particular, suppose $\pi: X_{1} \rightarrow X_{2}$ is a homomorphism $(\bmod 0)$ and each $X_{i}$ has been "topologized" (i.e., $\mathscr{F}_{i}$ is the Borel $\sigma$-field for some compact metric topology for which $T$ is a homeomorphism. We have seen any process is isomorphic $(\bmod 0)$ to some such topologized process). We can construct a new process $X_{1}^{\prime}$, isomorphic $(\bmod 0)$ to $X_{1}$, as follows: Let $\Omega_{1}^{\prime}=\Omega_{1} \times \Omega_{2}, \mathscr{F}_{1}^{\prime}=\mathscr{F}_{1} \times \mathscr{F}_{2}, T_{1}^{\prime}=T_{1} \times T_{2}$. Let $\pi_{1}(x)=(x, \pi x)$ and let $\mu_{1}^{\prime}=\mu_{1} \circ \pi_{1}^{-1}$. Then $X_{1}^{\prime}=\left(\Omega_{1}^{\prime}, \mathscr{F}_{1}^{\prime}, T_{1}^{\prime}, \mu_{1}^{\prime}\right)$ is isomorphic $(\bmod 0)$ to $X_{1}^{\prime}$ and the mapping $\pi^{\prime}\left(x_{1}, x_{2}\right)=x_{2}$ is a continuous surjective homomorphism of $X_{1}^{\prime}$ onto $X_{2}$, and moreover $\pi^{\prime} \circ \pi_{1}=\pi$ a.e. Results of this sort were obtained by Furstenberg [4, Appendix to $\S 1$ ] by the theory of representation of $C^{*}$ algebras.

If $X$ is a process then the transformation $T$ induces an isometry on $L_{2}(\Omega)$ (in fact on all $L_{p}$ spaces) by $f \rightarrow f \circ T$. We will denote this operator also by $T$, and so write 
$T f$ or $f \circ T$ interchangeably. Thus the complex number $\lambda$ is an eigenvalue if there is a square-integrable $\mu$-measurable function $f$ such that $f \circ T=\lambda f$ a.e. By $\mu$-measurable we mean measurable with respect to the $\mu$ completion of $\mathscr{F}$. In fact, a $\mu$-measurable function $f$, if it exists, can be replaced (see [13, p. 225, Problem 12]) by an $\mathscr{F}$ measurable function $f^{\prime}$ such that $f^{\prime}=f$ a.e. (and so $f^{\prime} \circ T=\lambda f^{\prime}$ a.e.). Note all eigenvalues are of modulus 1 . In fact, we could remove the a.e. qualification on the equality by setting $f^{\prime}$ equal to zero on an appropriate set but we will discuss a different approach shortly. A process $X$ is called ergodic in case the eigenvalue one has multiplicity exactly one. Alternatively, $X$ is ergodic if $T F=F, F \in \mathscr{F}$, implies $\mu(F) \cdot \mu(\Omega \mid F)=0$. If $X$ is ergodic and $\lambda$ is an eigenvalue with eigenfunction $f$, then $|f| \circ T=|f|$ a.e. and so $|f|$ is constant a.e. We can choose $f$ so that $|f|=1$ a.e. In general, we cannot make $|f|=1$ identically, for example if $\lambda \neq 1$ and there is some point $x$ such that $T x=x$. However, we can replace $X$ by an isomorphic $(\bmod 0)$ copy $X^{\prime}$, which then will have the same eigenvalues, such that each eigenvalue $\lambda$ admits an eigenfunction $f$ such that $|f|=1$ and $f \circ T=\lambda f$ identically.

It was pointed out in [4] that a $\lambda$-eigenfunction $f$ for $X$ can be regarded as a homomorphism (mod 0$)$ of $X$ onto the process of rotation by $\lambda(z \rightarrow \lambda z)$ on the unit circle, with the measure either atomic or Lebesgue depending on whether $\lambda$ is or is not a root of unity. We can treat the two cases just mentioned in a uniform manner by saying a $\lambda$-eigenfunction $f$ defines a homomorphism onto the process of translation on the compact dual group of the "discretized" group generated by $\lambda$. We will generalize this observation. Let $X$ be ergodic and let $\mathscr{P}(X)$ denote the set of eigenvalues for $X$. It is well known that $\mathscr{P}(X)$ is a subgroup of $\{|z|=1\}$. Let $\Lambda$ be any subgroup of $\mathscr{P}(X)$, endow $\Lambda$ with the discrete topology, and let $\Omega_{K}=\Lambda^{\prime}$, the compact dual of $\Lambda$. Since $\left(\Omega_{X}, \mathscr{F}_{X}\right)$ is standard Borel, $\Lambda$ is countable, and so $\Omega_{K}$ is metrizable. Let $\omega_{0}$ be that element of $\Omega$ such that $\omega_{0}(\lambda)=\lambda$ and let $T_{K}(\omega)=\omega+\omega_{0}$ where + is the group operation for $\Omega_{K}$. It is known (see [8, p. 47]) that eigenfunctions $f_{\lambda}$ can be selected so that $f_{\lambda_{1}} f_{\lambda_{2}}=f_{\lambda_{1} \lambda_{2}}$ a.e. and, since $\Lambda$ is countable, the exceptional null set can be chosen so that on the complement $f_{\lambda_{1}} f_{\lambda_{2}}=f_{\lambda_{1} \lambda_{2}}$ for all $\lambda_{1}, \lambda_{2}$. It is easy to verify that $x \rightarrow \hat{x}, \hat{x}(\lambda)=f_{\lambda}(x)$, defines a measurable mapping of $\Omega_{X}$ into $\Omega_{K}$ satisfying $(T x)^{\wedge}=T \hat{x}$ for $x$ not in the exceptional set. The measure $\mu_{X}$ is carried by this mapping into some invariant measure on $\Omega_{K}$. However, one easily verifies $\left(\Omega_{K}, T_{K}\right)$ is uniquely ergodic, with Haar measure $m$ the unique invariant measure, so $x \rightarrow \hat{x}$ is a homomorphism $(\bmod 0)$ from $X$ to $K=\left(\Omega_{K}, \mathscr{F}_{K}, m, T_{K}\right)$. This homomorphism is not quite canonical, since the choice of $\left\{f_{\lambda}\right\}$ is not canonical. However, if $\pi_{1}$ and $\pi_{2}$ are homomorphisms of $X$ into the Kronecker process $K$ (following Furstenberg, we call an ergodic translation on a compact metrizable group a Kronecker process) then the mapping $x \rightarrow \pi_{1}(x)-\pi_{2}(x)$ carries almost all of $X$ onto a single point $\omega_{1}$ in $K$. Thus, since translation by $\omega_{1}$ is an automorphism of the process $K$, any two homomorphisms $\pi_{1}$ and $\pi_{2}$ of $X$ into $K$ are related by an automorphism $\pi$ of $K, \pi_{1}=\pi \pi_{2}$.

Definition. If $X$ and $Y$ are ergodic processes and if $\Lambda=\mathscr{P}(X) \cap \mathscr{P}(Y)$ then the 
process $K$ constructed from $\Lambda$ as described above is called the maximum common Kronecker factor and is denoted $K(X, Y)$.

If $X$ and $Y$ are processes then $X \times Y$ denotes the product process $\left(\Omega_{X} \times \Omega_{Y}\right.$, $\left.\mathscr{F}_{X} \times \mathscr{F}_{Y}, \mu_{X} \times \mu_{Y}, T_{X} \times T_{Y}\right)$. The notation $X^{\wedge}$ will denote various forgetful functors in this paper. For now we mean to forget the measure, $X^{\wedge}=(\Omega, \mathscr{F}, T)$. If $X_{1}$ and $X_{2}$ are processes and $\pi: X_{1} \rightarrow X_{2}^{\wedge}$ is a homomorphism then a measure $\mu$ on $\left(\Omega_{1}, \mathscr{F}_{1}\right)$ is said to cover $\mu_{2}$ if $\mu \circ \pi^{-1}=\mu_{2}$. Thus the measure $\mu_{X} \times \mu_{Y}$ is an invariant measure on $(X \times Y)^{\wedge}$ which covers $\mu_{X}$ and $\mu_{Y}$ (with respect to the natural mappings). Processes $X$ and $Y$ are called disjoint if $\mu_{X} \times \mu_{Y}$ is the only invariant measure on $X^{\wedge} \times Y^{\wedge}$ which covers $\mu_{X}$ and $\mu_{Y}$. (It is not hard to see this is equivalent to the definition in [4].) We will frequently delete the "^^" when no misunderstanding is possible.

It is clear from our discussion of eigenfunctions as homomorphisms that if two ergodic processes have a common eigenvalue other than 1 then they have a nontrivial factor in common and so (see [4]) they cannot be disjoint. One purpose of this paper is to show that if $X$ is a Weyl process (see [4]) and if $Y$ is ergodic and if $X$ and $Y$ have no common eigenvalue other than 1, then $X$ and $Y$ are disjoint. In this section we will prove the more basic fact that if $X$ and $Y$ are ergodic and have no common eigenvalues, then $X \times Y$ is ergodic. Although the proof uses only typical spectral theory arguments, this fact is apparently not well known. For example, the fact that a weakly-mixing process and an ergodic process have an ergodic product, as proved in [4], is a corollary.

Let $X$ and $Y$ be (not necessarily ergodic) processes, and let $E_{X}$ and $E_{Y}$ be the spectral measures defined on the unit circle corresponding to the unitary operator $T_{X}$ and $T_{Y}$. (We will follow the notation and development of [9].) Let $E_{X}^{\prime}$ and $E_{Y}^{\prime}$ be the spectral measures corresponding to the transformations $T_{X} \times I_{Y}$ and $I_{X} \times T_{Y}$, where $I_{X}$ and $I_{Y}$ are the identity transformations on $X$ and $Y$ (really $X^{\wedge}$ and $Y^{\wedge}$ for a suitable forgetful functor). The transformations $T_{X} \times I_{Y}$ and $I_{X} \times T_{Y}$ commute, and so, as a rather direct consequence of Theorem 2 of [9], $E_{X}^{\prime}$ and $E_{Y}^{\prime}$ commute. It follows that we can construct a spectral measure $F$ defined on the Borel sets of the torus such that $F\left(M_{1} \times M_{2}\right)=E_{X}^{\prime}\left(M_{1}\right) E_{Y}^{\prime}\left(M_{2}\right)$ if $M_{1}$ and $M_{2}$ are Borel subsets of the circle. We omit the construction of $F$, but a similar construction of a planar product measure from commuting measures on the real line is carried out in [9, pp. 72-73], and a more general discussion is given in [1] under the title of amalgamation. Let $f$ be the mapping of the torus onto the circle given by $f((\lambda, \mu))$ $=\lambda \mu$ and set $E(M)=F\left(f^{-1}(M)\right)$ for Borel sets $M$. Clearly $E$ is a spectral measure on the circle satisfying $\int \nu d E(\nu)=\int \lambda \mu d F((\lambda, \mu))$.

THEOREM 1.1. $E$ is the spectral measure of $T_{X} \times T_{Y}$.

Proof. The assertion is equivalent to $\int \lambda \mu d(F(\lambda, \mu) h, h)=\left(T_{X} \times T_{Y} h, h\right)$ for each $h$ in $L_{2}(X \times Y)\left[9\right.$, p. 60]. We will instead show that $\int \lambda \mu d\left(F(\lambda, \mu) \alpha \beta, \alpha^{\prime} \beta^{\prime}\right)$ $=\left(T_{X} \times T_{Y} \alpha \beta, \alpha^{\prime} \beta^{\prime}\right)$ for square integrable $\alpha(x), \alpha^{\prime}(x), \beta(y), \beta^{\prime}(y)$. The proposition then follows by linearity and continuity. 
Let $m(M)=\left(F(M) \alpha \beta, \alpha^{\prime} \beta^{\prime}\right), \quad m_{1}\left(M_{1}\right)=\left(E_{X}\left(M_{1}\right) \alpha, \alpha^{\prime}\right), \quad m_{2}\left(M_{2}\right)=\left(E_{Y}\left(M_{2}\right) \beta, \beta^{\prime}\right)$ where $M$ is a Borel subset of the torus and $M_{1}$ and $M_{2}$ are Borel subsets of the circle. If $M=M_{1} \times M_{2}$ then $F\left(M_{1} \times M_{2}\right)=E_{X}^{\prime}\left(M_{1}\right) E_{Y}^{\prime}\left(M_{2}\right)$. A key observation is that $E_{X}^{\prime}\left(M_{1}\right) \alpha \beta=E_{X}(\alpha) \beta$. To see this, let $\alpha=\alpha_{0}, \alpha_{1}, \alpha_{2}, \ldots$ and $\beta=\beta_{0}, \beta_{1}, \beta_{2}, \ldots$ be normal bases for $L_{2}(X)$ and $L_{2}(Y)$ respectively. Then $\left\{\alpha_{i} \beta_{j}\right\}$ is a normal basis for $L_{2}(X \times Y)$ and the equation $P\left(M_{1}\right) \alpha_{i} \beta_{j}=\left(E_{X}\left(M_{1}\right) \alpha_{i}\right) \beta_{j}$ determines a projection valued measure $P$ for which $\int \lambda d P=T_{X} \times I_{Y}$. By uniqueness of the spectral resolution, $P=E_{X}^{\prime}$. Since $P\left(M_{1}\right) \alpha \beta=E_{X}(\alpha) \beta$ by definition, the observation is justified. A similar result holds for $E_{Y}^{\prime}$, and we conclude that $F\left(M_{1} \times M_{2}\right) \alpha \beta$ $=\left(E_{X}\left(M_{1}\right) \alpha\right)\left(E_{Y}\left(M_{2}\right) \beta\right)$, and so $m\left(M_{1} \times M_{2}\right)=\left(\left(E_{X}\left(M_{1}\right) \alpha\right)\left(E_{Y}\left(M_{2}\right) \beta\right), \alpha^{\prime} \beta^{\prime}\right)$. By Fubini's Theorem, this is $\left(E_{X}\left(M_{1}\right) \alpha, \alpha^{\prime}\right)\left(E_{Y}\left(M_{2}\right) \beta, \beta^{\prime}\right)=m_{1}\left(M_{1}\right) m_{2}\left(M_{2}\right)$. Thus $m=m_{1} \times m_{2}$. Fubini's Theorem, now applied to $m$, implies

$$
\begin{aligned}
\int \lambda \mu d\left(F(\lambda, \mu) \alpha \beta, \alpha^{\prime} \beta^{\prime}\right) & =\int \lambda \mu d m(\lambda, \mu)=\int \lambda d m_{1}(\lambda) \int \mu d m_{2}(\mu) \\
& =\int \lambda d\left(E_{X}(\lambda) \alpha, \alpha^{\prime}\right) \int \mu d\left(E_{Y}(\mu) \beta, \beta^{\prime}\right) \\
& =\left(T_{X} \alpha, \alpha^{\prime}\right)\left(T_{Y} \beta, \beta^{\prime}\right)
\end{aligned}
$$

which is, again by Fubini's Theorem, $\left(T_{X} \times T_{Y} \alpha \beta, \alpha^{\prime} \beta^{\prime}\right)$. Q.E.D.

COROLlaRY 1.2. Let $X$ and $Y$ be processes. Let $\lambda$ be in the point spectrum $\mathscr{P}(X \times Y)$ and $\left(T_{X} \times T_{Y}\right) h=\lambda h$. Then there is a collection of numbers $C_{\mu \nu}, \mu \in \mathscr{P}(X)$ and $\nu \in \mathscr{P}(Y)$, and eigenfunctions $f_{\mu}(x), g_{\nu}(y)$, corresponding to $\mu, \nu$, such that $h(x, y)=\sum_{\mu \nu=\lambda} C_{\mu \nu} f_{\mu}(x) g_{\nu}(y)$. In particular, $\mathscr{P}(X \times Y)=\mathscr{P}(X) \cdot \mathscr{P}(Y)$.

Proof. It suffices to show that if $h \in L_{2}(X \times Y)$ satisfies $\left(T_{X} \times T_{Y}\right) h=\lambda h$ and $\left(h, f_{\mu} g_{\nu}\right)=0$ whenever $\mu \nu=\lambda$ and $T_{X} f_{\mu}=\mu f_{\mu}, T_{Y} f_{v}=\nu g_{v}$, then $h=0$. Since $h$ is a $\lambda$-eigenfunction, $h=0$ if and only if $E(\{\lambda\}) h=0$. The orthogonality assumptions on $h$ imply that $h$ is a sum (perhaps infinite) of functions of the form $f(x) g(y)$, with $\left(f g, f_{\mu} g_{v}\right)=0$ whenever $f_{\mu}, g_{v}$ are as above. It will suffice to show $\left(E(\{\lambda\}) f g, f_{\mu} g_{v}\right)=0$. But the proof of the preceding proposition showed that the measure $\left(E(\cdot) f g, f_{\mu} g_{v}\right)$ is the convolution of the measures $\left(E_{X}(\cdot) f, f_{\mu}\right)$ and $\left(E_{Y}(\cdot) g, g_{v}\right)$ (in the notation of the preceding proposition, the measure is $m \circ f^{-1}$ which is the definition of $\left.m_{1} * m_{2}\right)$. The atoms of $E_{X}$ occur at points of $\mathscr{P}(X)$ and, since $f_{\mu}$ is a $\mu$-eigenfunction, $\left(E_{X}(\cdot) f, f_{u}\right)$ has (at most) a single atom at $\mu$. Similarly, $\left(E_{Y}(\cdot) g, g_{v}\right)$ has an atom only at $\nu$. But $\left(f, f_{\mu}\right)\left(g, g_{v}\right)=\left(f g, f_{\mu} g_{v}\right)=0$ so either $\left(f, f_{u}\right)$ or $\left(g, g_{v}\right)$ is zero and so either $\left(E_{X}(\mu) f, f_{\mu}\right)$ or $\left(E_{Y}(v) g, g_{v}\right)$ is zero. Thus one of the two factors in the convolution is nonatomic, and so the product is nonatomic. It follows, as desired, that $\left(E(\{\lambda\}) f g, f_{\mu} g_{v}\right)=0$.

As for the last remark in the statement of the corollary, $f_{\mu} g_{\nu}$ has eigenvalue $\mu \nu$ so $\mathscr{P}(X \times Y) \supseteq \mathscr{P}(X) \cdot \mathscr{P}(Y)$ and the above reasoning shows $\mathscr{P}(X \times Y) \subseteq \mathscr{P}(X)^{\circ} \cdot \mathscr{P}(Y)$. Q.E.D. 
The following corollary is now obvious:

Corollary 1.3. $X \times Y$ is ergodic if and only if $X$ and $Y$ are ergodic and $\mathscr{P}(X) \cap \mathscr{P}(Y)=\{1\}$.

J. Auslander has pointed out in a private communication that if processes $X$ and $Y$ are disjoint then at least one of them is ergodic. We will study pairs of processes $X$ and $Y$ where both are ergodic. The fact, mentioned earlier, that disjointness of such $X$ and $Y$ implies $X \times Y$ is ergodic causes difficulties in the study of disjointness. In the next section we will develop a more general notion, quasi-disjointness, which does not imply an ergodic product but reduces to disjointness in the case of an ergodic product.

2. Quasi-disjointness. In the following proposition and theorem, we will see that in the case where the point spectra of ergodic processes $X$ and $Y$ overlap, the maximum common Kronecker factor has a close relationship to the ergodic decomposition of the measure $\mu_{X} \times \mu_{Y}$. We will use certain results of [3], and these results are stated for locally compact spaces and $\mu$-proper mappings. But a standard Borel space admits a compact metric topology (either that of the unit interval, or that of a compact subset of $\left.\left\{0,1, \frac{1}{2}, \frac{1}{3}, \ldots\right\}\right)$ and $\mu$-proper reduces to what we have called $\mu$-measurable.

Proposition 2.1. Let $X$ and $Y$ be ergodic processes, $K=K(X, Y)$, let $\alpha: X \rightarrow K$ and $\beta: Y \rightarrow K$ be homomorphisms (mod 0$)$ and let $\gamma(x, y)=\alpha(x)-\beta(y)$. Then $\gamma$ is $a$ homomorphism $(\bmod 0)$ of $X \times Y$ to $\left(\Omega_{K}, \mathscr{F}_{K}, \nu, I\right)$ where $I$ is the identity mapping and $\nu=\mu_{X} \times \mu_{Y} \circ \gamma^{-1}$. Moreover, if $f$ is a square-integrable $\mu_{X} \times \mu_{Y}$-measurable function on $X \times Y$ satisfying $f \circ T_{X} \times T_{Y}=f$ a.e. then there is a v-measurable and square-integrable function $f^{\prime}$ on $\Omega_{K}$ such that $f^{\prime} \circ \gamma=f$ a.e. In particular, if $F$ is a $\mu_{X} \times \mu_{Y}$-measurable subset of $X \times Y$ such that $T_{X} \times T_{Y}(F) \Delta F$ is of measure zero then there is a $\nu$-measurable subset $F^{\prime}$ of $\Omega_{K}$ such that $F \Delta \gamma^{-1}\left(F^{\prime}\right)$ is of measure zero.

Proof. We know that $\alpha$ and $\beta$ can be restricted to invariant subsets of measure one such that the restricted mappings are homomorphisms (not just (mod 0)) of their domains. It follows that $\gamma$ is $\mu_{X} \times \mu_{Y}$-measurable and for a subset of measure one,

$$
\gamma\left(T_{X} x, T_{Y} y\right)=\alpha\left(T_{X} x\right)-\beta\left(T_{Y} y\right)=T_{K}(\alpha(x))-T_{K}(\beta(y))=\alpha(x)-\beta(y)=\gamma(x, y) .
$$

Since $\nu$ is defined as $\mu_{X} \times \mu_{Y} \circ \gamma^{-1}$, the stated homomorphism property of $\gamma$ follows.

Now let $f$ be as described above. From Corollary 1.2 it follows that $f=\sum C_{\lambda} F_{\lambda} G_{\lambda}{ }^{-1}$ where $F_{\lambda}$ is a $\lambda$-eigenfunction on $X$ and $G_{\lambda}-1$ is a $\lambda^{-1}$-eigenfunction on $Y$. Since $\mathscr{P}(X)$ and $\mathscr{P}(Y)$ are groups, it follows that the $\lambda$ 's involved are in $\mathscr{P}(X) \cap \mathscr{P}(Y)$. For each such $\lambda$ there is a unique character $\chi_{\lambda}$ on $K$ such that $T_{K} \chi_{\lambda}=\lambda \chi_{\lambda}$, and the set $\left\{\chi_{\lambda}\right\}$ is such that $\chi_{\lambda_{1} \lambda_{2}}=\chi_{\lambda_{1}} \chi_{\lambda_{2}}$ (in particular $\chi_{\lambda}-1=\left(\chi_{\lambda}\right)^{-1}$ ). Clearly $F_{\lambda}(x)=\chi_{\lambda} \alpha(x)$ and $G_{\lambda}{ }^{-1}(y)=\chi_{\lambda^{-1}} \beta(y)$ are $\lambda$-eigenfunctions. Since $\chi_{\lambda}$ is a 
character, $\chi_{\lambda}(\alpha(x)) \chi_{\lambda}^{-1}(\beta(y))=\chi_{\lambda}(\alpha(x)-\beta(y))=\chi_{\lambda}(\gamma(x, y))$. We now choose $C_{\lambda}$ so that $f=\sum C_{\lambda} F_{\lambda} G_{\lambda}$ and set $f^{\prime}=\sum C_{\lambda} \chi_{\lambda}$. This establishes the claim for $f$. The assertion about the set $F$ follows from applying the above to the characteristic function of $F$. Q.E.D.

We continue using the notation of the preceding proposition. It follows from Theorem 1 of Chapter $6, \S 3$, No. 1 of [3] that there is a mapping $k \rightarrow \mu_{k}$ from $\Omega_{K}$ into the set of probability measures on $\Omega_{X} \times \Omega_{Y}$ which "disintegrates" $\mu_{X} \times \mu_{Y}$ with respect to $\gamma$. That is, $\mu_{k}\left(\gamma^{-1}(k)\right) \equiv 1$ and for each measurable $F$ in $X \times Y$ the function $k \rightarrow \mu_{k}(F)$ is a $\nu$-measurable function satisfying $\int \mu_{k}(F) d \nu(k)=\mu_{X} \times \mu_{Y}(F)$. We write $\mu_{X} \times \mu_{Y}=\int \mu_{k} d \nu(k)$. Moreover, if $\mu_{k}^{\prime}$ is any other disintegration of $\mu_{X} \times \mu_{Y}$ then $\mu_{k}=\mu_{k}^{\prime}$ for $\nu$-almost all $k$.

THEOREM 2.2. Let $\mu_{k}$ be a $\gamma$-disintegration of $\mu_{X} \times \mu_{Y}$ as described above. Then there is a $\nu$-null set $N$ in $K$ such that for each $k$ not in $N, \mu_{k}$ is a $T_{X} \times T_{Y}$-ergodic and invariant measure which covers both $\mu_{X}$ and $\mu_{Y}$.

Proof. The invariance of the $\mu_{k}$ is a simple consequence of the essential uniqueness of the disintegration of $\mu_{X} \times \mu_{Y}$ and the invariance of the sets $\gamma^{-1}(k)$. For clearly the family $\mu_{k} \circ\left(T_{X} \times T_{Y}\right)$ is a disintegration of $\mu_{X} \times \mu_{Y}$, so $\mu_{k} \circ T_{X} \times T_{Y}=\mu_{k}$ a.e.

For brevity set $t=(x, y)$ and $T=T_{X} \times T_{Y}$. The mapping $t \rightarrow \mu_{\gamma(t)}$ is such that $\mu_{X} \times \mu_{Y}=\int \mu_{\gamma(t)} d \mu_{X} \times \mu_{Y}(t)$ and, for almost all $t, \mu_{\gamma(T t)}=\mu_{\gamma(t)}$ as well as $\mu_{\gamma(t)} T=\mu_{\gamma(t)}$. From the work of Varadarajan (Theorem 4.2 of [14]) or, since we are dealing with a single transformation, from the earlier work of Blum and Hanson [2], we know that there is a mapping $t \rightarrow \beta_{t}$ from $X \times Y$ into the $T$-invariant ergodic probability measures on $X^{\wedge} \times Y^{\wedge}$ such that $\beta_{T t}=\beta_{t}$ for all $t, t \rightarrow \beta_{t}(F)$ is measurable for any measurable $F$, for such $F$ and any $T$-invariant measure $\mu$ (such as $\left.\mu_{X} \times \mu_{Y}\right) \mu(F)$ $=\int \beta_{t}(F) d \mu(t)$, and if $\beta$ is any $T$-invariant ergodic measure then $\beta\left(\left\{t \mid \beta_{t_{o}}=\beta\right\}\right)=1$. We will show that $\mu_{\gamma(t)}=\beta_{t}$ for $\mu_{X} \times \mu_{Y}$-almost all $t$, from which it follows readily that almost all $\mu_{k}$ are ergodic.

It is known that if we impose the weak measure structure on the collection $E_{1}$ of ergodic measures (i.e., the structure defined by requiring the functions $\mu \rightarrow \mu(F)$ to be measurable for each measurable $F$ ) then the resulting measurable space is a standard Borel space (see the remarks following Definition 2.4). In the case that this space is isomorphic to the unit interval, the previous proposition implies there is a mapping $k \rightarrow \mu_{k}^{\prime}$ of $K$ into $E_{1}$ such that $\beta_{t}=\mu_{\gamma(t)}^{\prime}$ a.e. One easily verifies that $\mu_{k}^{\prime}$ must then be a $\gamma$-disintegration of $\mu_{X} \times \mu_{Y}$, and so $\mu_{\gamma(t)}^{\prime}=\mu_{\gamma(t)}$ a.e. The other alternative for $E_{1}$ is that it be countable. By identifying each point in $E_{1}$ with an interval in $[0,1]$ of appropriate length, we can apply the same argument just given. Q.E.D.

Definition 2.3. Let $X, Y, K, \alpha, \beta, \gamma$, and $\nu$ be as above. We say $X$ and $Y$ are quasi-disjoint if for $\nu$-almost all $k$ there is exactly one ergodic measure $\mu=\mu_{K}$ such that $\mu \circ T_{X} \times T_{Y}=\mu, \mu$ covers $\mu_{X}$ and $\mu_{Y}$, and $\mu\left(\gamma^{-1}(k)\right)=1$.

Remark 1. Suppose $\alpha^{\prime}: X \rightarrow K, \beta^{\prime}: Y \rightarrow K$ are possibly different homomorphisms $(\bmod 0)$ and $\gamma^{\prime}(x, y)=\alpha^{\prime}(x)-\beta^{\prime}(y)$. We have observed $\alpha^{\prime}$ is the composition 
of $\alpha$ and a translation on $\Omega_{k}$, a similar remark applies to $\beta^{\prime}$ and $\beta$, and so there is a $k^{\prime}$ such that $\gamma^{\prime}(x, y)=\gamma(x, y)+k^{\prime}$ a.e. Let $N$ be a $\nu$-null set such that $k \notin N$ implies $\gamma^{-1}(k)$ supports exactly one invariant covering measure. Let $N^{\prime}=k^{\prime}+N$ and $\nu^{\prime}=\mu_{X} \times \mu_{Y} \circ\left(\gamma^{\prime}\right)^{-1}$. One easily checks that $\left(\gamma^{\prime}\right)^{-1}\left(N^{\prime}\right)=\gamma^{-1}(N)$, so that $N^{\prime}$ is a $\nu^{\prime}$-null set. Moreover, if $k \notin N^{\prime}$ then $\left(\gamma^{\prime}\right)^{-1}(k)=\gamma^{-1}\left(k-k^{\prime}\right)$ and $k-k^{\prime} \notin N$, so the set $\left(\gamma^{\prime}\right)^{-1}(k)$ supports exactly one invariant covering measure. This shows that the notion of quasi-disjointness is independent of the choice of homomorphisms $(\bmod 0) \alpha$ and $\beta$. It follows readily from this that quasi-disjointness is also insensitive to replacing $X$ and $Y$ by isomorphic $(\bmod 0)$ copies $X^{\prime}$ and $Y^{\prime}$.

REMARK 2. Since $X \times Y$ is ergodic if and only if $K$ is trivial, in which case the definition of quasi-disjointness reduces to that of disjointness, and since the product of disjoint ergodic processes is ergodic, we see $X$ and $Y$ are disjoint if and only if $X$ and $Y$ are quasi-disjoint and have an ergodic product. In particular, if either $X$ or $Y$ is weakly mixing, quasi-disjointness is equivalent to disjointness. We also observe that if $X$ and $Y$ are quasi-disjoint and have no nontrivial factors in common, then $X$ and $Y$ are disjoint (since $X$ and $Y$ must have no common eigenvalues other than 1).

EXAmPle 1. Suppose $X$ and $Y$ are Kronecker processes. Since Kronecker processes with the same spectrum are isomorphic, we can suppose $\Omega_{X}$ is the dual group to $\mathscr{P}(X)$, the point spectrum of $X$, and that $T_{X}(x)=x+x_{0}$ where $x_{0}(\lambda)=\lambda$. A similar supposition is made for $Y$. The maximum common Kronecker factor $K$ is also a translation on a group, the dual group of $\Lambda=\mathscr{P}(X) \cap \mathscr{P}(Y)$. Let $\alpha: X \rightarrow K$ and $\beta: Y \rightarrow K$ be the restriction mappings, i.e., $\alpha(x)(\lambda)=x(\lambda)$ for $\lambda$ in $\Lambda$. One can easily verify that $\gamma(x, y)=\alpha(x)-\beta(y)$ is a group homomorphism of $\Omega_{X} \times \Omega_{Y}$ onto $\Omega_{K}$ and that $\gamma\left(x_{0}, y_{0}\right)=e=$ identity. Observe $T_{X} \times T_{Y}$, restricted to $\gamma^{-1}(e)$, is a translation on a compact Abelian group. One can check that for each $\left(\lambda_{1}, \lambda_{2}\right)$ in $\mathscr{P}(X)$ $\times \mathscr{P}(Y)$, the equation $\left(\lambda_{1}, \lambda_{2}\right)^{\prime}\left(k_{1}, k_{2}\right)=\lambda_{1}\left(k_{1}\right) \lambda_{2}\left(k_{2}\right)^{-1}$ defines a character $\left(\lambda_{1}, \lambda_{2}\right)^{\prime}$ on $\gamma^{-1}(e)$, that every character on $\gamma^{-1}(e)$ is of this form, and that $\left(\lambda_{1}, \lambda_{2}\right)^{\prime} \circ T_{X} \times T_{Y}$ $=\lambda_{1} \lambda_{2}^{-1} \cdot\left(\lambda_{1}, \lambda_{2}\right)^{\prime}$. Different characters $\left(\lambda_{1}, \lambda_{2}\right)^{\prime}$ on $\gamma^{-1}(e)$ have different eigenvalues $\lambda_{1} \lambda_{2}^{-1}$, so $\left(\gamma^{-1}(e), T_{X} \times T_{Y}\right)$ is uniquely ergodic. In particular, $\gamma^{-1}(e)$ supports only one invariant covering measure. By employing a suitable translation, one can verify that each $\gamma^{-1}(k)$ supports a unique invariant covering measure. We have shown that any two Kronecker processes are quasi-disjoint. In the next example, we will see that the exceptional set of $k$ for which $\gamma^{-1}(k)$ does not admit a unique invariant covering measure may not be void.

EXAMPLE 2. Let $\Omega_{X}$ be the two-dimensional torus,

$$
x=\left(\begin{array}{l}
x_{1} \\
x_{2}
\end{array}\right) \in \Omega_{X}, \quad T_{X} x=\left(\begin{array}{ll}
1 & 1 \\
0 & 1
\end{array}\right)\left(\begin{array}{l}
x_{1} \\
x_{2}
\end{array}\right)+\left(\begin{array}{l}
u \\
v
\end{array}\right)
$$

with arithmetic performed modulo 1 . We choose $v$ to be irrational. From the work of F. Hahn [5, Theorem 8 and Theorem 18] we conclude $T$ is strictly ergodic on $\Omega$, and clearly Haar measure is the unique invariant measure. An easy com- 
putation with exponentials shows that translation by $v(\bmod 1)$ on $[0,1)$ is the maximum Kronecker factor. We let $Y=X$,

$$
\alpha\left(\begin{array}{l}
x_{1} \\
x_{2}
\end{array}\right)=x_{2}, \quad \beta\left(\begin{array}{l}
y_{1} \\
y_{2}
\end{array}\right)=y_{2}
$$

and

$$
\gamma\left(\left(\begin{array}{l}
x_{1} \\
x_{2}
\end{array}\right),\left(\begin{array}{l}
y_{1} \\
y_{2}
\end{array}\right)\right)=x_{2}-y_{2}
$$

For $k$ on $[0,1)$ the transformation on $\gamma^{-1}(k)$ can be given as $\left(x_{1}, x_{2}, y_{1}, y_{2}\right)$ $\rightarrow\left(x_{1}+x_{2}+u, x_{2}+v, y_{1}+x_{2}+u-k, x_{2}-k+v\right)$ where $y_{2}$ has been replaced by $x_{2}-k$. Since the second coordinate $x_{2}$ determines the fourth coordinate $y_{2}=x_{2}-k$, this transformation can be represented on the three-dimensional torus as $(x, y, z)$ $\rightarrow(x+y+u, y+v, y+z+u-k)$, i.e., as an affine transformation with the automorphism given by

$$
A=\left(\begin{array}{lll}
1 & 1 & 0 \\
0 & 1 & 0 \\
0 & 1 & 1
\end{array}\right)
$$

and the translation part given by

$$
\left(\begin{array}{c}
u \\
v \\
u-k
\end{array}\right)
$$

Now any invariant measure on $\gamma^{-1}(k)$ will project onto an invariant measure on $X$ (and $Y$ ) and so, since $X$ is uniquely ergodic, it will be a covering measure. Thus $\gamma^{-1}(k)$ will support a unique invariant covering measure if and only if it is uniquely ergodic (under $T_{X} \times T_{Y}$ ). We work with the three-dimensional representation given above. Let $A^{*}$ be the adjoint of $A$, observe $\left(A^{*}-I\right)^{2}=0$ and $\operatorname{ker}\left(A^{*}-I\right)$ $=\{(s, t,-s)\}$. It follows from the above cited theorems of Hahn that the transformation is ergodic with respect to Haar measure if and only if it is strictly ergodic, and that this happens if and only if $s u+t v+(-s)(u-k) \equiv 0(\bmod 1)$ has no solutions in integers $s$ and $t$. This is clearly equivalent to the assertion that $1, v$, and $k$ be rationally independent. It is easy to see that the measure $\nu=\mu_{X} \times \mu_{Y} \circ \gamma^{-1}$ is Haar measure, so the countable set of $k$ which depend rationally on 1 and $v$ is of measure zero. Thus $X$ is quasi-disjoint from itself.

The above example is also of interest from the point of view of topological dynamics. One could imagine an analogous notion of quasi-disjointness, with the maximal Kronecker factor replaced by the maximal equicontinuous factor, and with the requirement that $\gamma^{-1}(k)$ support a unique invariant covering measure replaced by the requirement that $\gamma^{-1}(k)$ be minimal. When $k$ is rationally independent from 1 and $v$, the transformation is minimal, as follows from the work of Hahn and Parry [6, see particularly Theorem 7]. Thus $\gamma^{-1}(k)$ is minimal, except for a set of $K$ of first category. 
Definition 2.4. Let $Q$ consist of all ergodic processes which are quasi-disjoint from every ergodic process.

Let $X, Y, K$ and $\gamma$ and $\nu$ continue to have the meaning given to them above. Let $E_{1}=E_{1}(X, Y)$ be the set of invariant probability measure on $X^{\wedge} \times Y^{\wedge}$ and let $E \subseteq E_{1}$ consist of those measures which moreover cover $\mu_{X}$ and $\mu_{Y}$. We let $\mathscr{E}$ be the smallest $\sigma$-algebra of subsets of $E_{1}$ such that $\mu \rightarrow \mu(F)$ is measurable for each measurable $F$. It is known that $\left(E_{1}, \mathscr{E}\right)$ is a standard Borel space [14, Theorem 4.2] and a simple argument using countable generating algebras for $\mathscr{F}_{X}$ and $\mathscr{F}_{Y}$ and the monotone class theorem shows that $E \in \mathscr{E}$. Recall that $\gamma(x, y)=\alpha(x)-\beta(y)$ is defined only on a measurable rectangle of full measure. Let $N$ be the complement of the domain of definition. One easily sees that if $\mu$ is in $E$, then $\mu(N)=0$, so $\mu \circ \gamma^{-1}$ is a probability measure on $\Omega_{K}$. The fact that $\mu$ is ergodic and $\gamma$ is invariant implies $\mu \circ \gamma^{-1}$ is concentrated at a point $k$. We set $f(\mu)=k$. If $F$ is a measurable subset of $\Omega_{K}$ then $f^{-1}(F)=E \cap\left\{\mu \mid \mu\left(\gamma^{-1}(F)\right)=1\right\}$ which shows that $f$ is measurable. From Theorem 4.4 of [14] we know there is a unique measure $\sigma$ on $E_{1}$ such that for any $F$ in $\mathscr{F}_{X} \times \mathscr{F}_{Y}, \mu_{X} \times \mu_{Y}(F)=\int_{E_{1}} \mu(F) d \sigma(\mu)$. The definitions of the objects involved imply that $\sigma(E)=1$ and, for a measurable set $F, \sigma\left(f^{-1}(F)\right)=\int_{f^{-1}(F)} 1 d \sigma(\mu)$ $=\int_{E} \mu\left(\gamma^{-1}(F)\right) d \sigma(\mu)=\mu_{X} \times \mu_{Y}\left(\gamma^{-1}(F)\right)=\nu(F)$, i.e., $\sigma \circ f^{-1}=\nu$. The following obvious proposition and subsequent remark provides us with a revealing formulation of quasi-disjointness.

Proposition 2.5. The ergodic processes $X$ and $Y$ are quasi-disjoint if and only if there is a $\nu$-null set $N$ in $\Omega_{K}$ such that $f$, when restricted to $E \cap f^{-1}(N)$, is one-to-one.

REMARK. The mapping $f$ is always one-to-one $(\bmod 0)$ in the sense that a $\sigma$ null set $N^{\prime}$ can be deleted from $E$ such that $f$ is one-to-one on the residual. The difference between this assertion and that of the proposition can be seen clearly by choosing $X$ and $Y$ to be weakly mixing processes. Quasi-disjointness of $X$ and $Y$ in this case would imply the existence of a unique invariant measure (which is, of course, generally false).

3. Properties of the class $Q$. In this section we will show that $Q$ is closed under ergodic group extensions and passage to factors. In particular $Q$ contains the class of Weyl processes as defined in [4].

It is frequently possible to obtain information about a process $X$ by identifying certain "symmetric" points and lifting information from the identification space. By a symmetry of a process $X$ we mean a bimeasurable bijection $S$ of $\Omega_{X}$ to itself such that $T \circ S=S \circ T$ and $\mu_{X} \circ S=\mu_{X}$. We suppose $G$ is a group with a representation in the group of symmetries of $X$. We write the action of $G$ on $X$ as multiplication, $(g, x) \rightarrow g x$. In order to study quasi-disjointness, we make certain measurability assumptions. We suppose that $G$ is a standard Borel group [11], i.e., that there is a $\sigma$-algebra $\mathscr{F}_{G}$ such that $\left(G, \mathscr{F}_{G}\right)$ is a standard Borel space and $\left(g_{1}, g_{2}\right)$ $\rightarrow g_{1} g_{2}^{-1}$ is measurable. We also suppose there is a probability measure $m$ on $G$ which is left invariant. It follows from Theorem 7.1 of [11] that $m$ is also right 
invariant. Indeed, the cited theorem states that $\left(G, \mathscr{F}_{G}\right)$ can be regarded as a locally compact group. Since a right translate of $m$ is a left invariant probability measure it must be $m$. We assume also that $(g, x) \rightarrow g x$ is jointly measurable. It follows from Fubini's Theorem that $(\mu, g) \rightarrow \mu(g F)$ is jointly measurable, where $\mu$ belongs to the set of ergodic invariant measures with the weak measurability structure discussed earlier. Our final restriction on the action is, roughly, that the identification space should be a standard Borel space $(\bmod 0)$. That is, we assume there is a process $Y$ and a homomorphism $\pi: X \rightarrow Y$ (not necessarily surjective) such that $\pi x_{1}=\pi x_{2}$ if and only if $x_{1} \in G x_{2}$ (see Theorem 5.2 of [11] for a criterion for existence of $Y$ ). We will then say $X$ is a $G$-extension of $Y$, or $Y$ is a $G$-reduction of $X$. This notion was developed in [4], with a somewhat less extensive discussion of the assumptions on the action.

Theorem 3.1. Let $X, Y$ and $Z$ be ergodic processes, and suppose $X$ is a $G$ extension of $Y$. If $Y$ and $Z$ are quasi-disjoint, then $X$ and $Z$ are quasi-disjoint.

REMARK. The quasi-disjointness occurring in both examples of $\S 2$ can be deduced from the above theorem.

Proof of the theorem. We let $K=K(X, Z), \alpha: X \rightarrow K, \beta: Z \rightarrow K, \gamma(x, z)=\alpha(x)$ $-\beta(z), \nu=\mu_{X} \times \mu_{Z} \circ \gamma^{-1}$, and let 'denote the analogous objects for $Y$ and $Z$, e.g., $K^{\prime}=K(Y, Z)$. We suppose $\pi_{1}: X \rightarrow Y$ is the homomorphism involved in the definition of a group extension and set $\pi(x, z)=\left(\pi_{1} x, z\right)$. We let $\pi_{K}: \Omega_{K} \rightarrow \Omega_{K^{\prime}}$ be the restriction mapping, $\pi_{K}(k)(\lambda)=k(\lambda)$ for $\lambda$ in $\mathscr{P}(Y) \cap \mathscr{P}(Z)$. We omit the simple argument which shows that for proper choices of $\alpha, \beta, \alpha^{\prime}$ and $\beta^{\prime}$ the diagram below commutes:

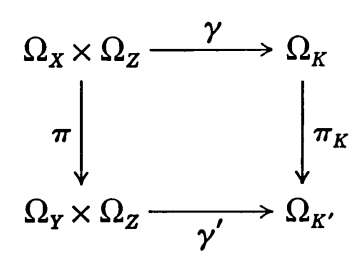

We let $N$ and $N^{\prime}$ be null sets in $\Omega_{X} \times \Omega_{Y}$ and $\Omega_{Y} \times \Omega_{Z}$ such that $\gamma(T x, T z)=\gamma(x, z)$, $\gamma^{\prime}(T y, T z)=\gamma^{\prime}(y, z)$ on the complements of $N$ and $N^{\prime}$. Let $E$ consist of the invariant measures on $X^{\wedge} \times Z^{\wedge}$ which cover $\mu_{X}$ and $\mu_{Z}$, and let $E^{\prime}$ be the corresponding object for $Y$ and $Z$.

We should like to caution the reader against dismissing the null sets $N$ and $N^{\prime}$ as if they were void, as $G \cdot N$ may be all of $\Omega$. It is easy to see, however, that $N$ and $N^{\prime}$ can be chosen so that $N \supseteq \pi^{-1}\left(N^{\prime}\right)$. For any measure $\mu$ in $E$ we let $\bar{\mu}(F)$ $=\int_{G} \mu(g F) d m(g)$. Since $\pi^{-1}\left(N^{\prime}\right)$ is $G$-invariant, $\vec{\mu} \circ \pi^{-1}\left(N^{\prime}\right)=0$. It is easy to check that $\bar{\mu} \circ \pi^{-1}$ is an ergodic invariant covering measure, so it is in $E^{\prime}$. Moreover, $\pi_{K} f(\mu)=f^{\prime}(\bar{\mu})$ where $f$ is the function from $E$ to $\Omega_{K}$ such that $\gamma^{-1} f(\mu)$ supports $\mu$. Using the quasi-disjointness of $Y$ and $Z$ and the easily checked fact that $\nu \circ \pi_{K}^{-1}$ 
$=\nu^{\prime}$, we conclude that for $\nu$-almost any $k$, if $\mu_{1}$ and $\mu_{2}$ are in $E^{\prime}$ and supported by $\gamma^{-1}(k)$ then $\bar{\mu}_{1} \circ \pi^{-1}=\bar{\mu}_{2} \circ \pi^{-1}$. The measures $\bar{\mu}_{1}$ and $\bar{\mu}_{2}$ are then $G$-invariant measures agreeing on $G$-invariant sets, and from a theorem of Varadarajan [14, Theorem 5.1], $\bar{\mu}_{1}=\bar{\mu}_{2}$.

Consider now the ergodic disintegration $\mu_{k}$ of $\mu_{X} \times \mu_{Y}$. Thus $f\left(\mu_{k}\right)=k$ and $\int \mu_{k} d \nu(k)=\mu_{X} \times \mu_{Y}$. We observe $\iint \mu_{k} \circ g d \nu(k) d m(g)=\int \mu_{X} \times \mu_{Y} \circ g d m(g)=\mu_{X} \times \mu_{Y}$. Now if $k \rightarrow \mu_{k}^{\prime}$ is another measurable mapping of $\Omega_{K}$ into $E$ satisfying $f\left(\mu_{k}^{\prime}\right)=k$ then, by what we have just seen, $\int \mu_{k} \circ g d m(g)=\int \mu_{k}^{\prime} \circ g d m(g)$ and so, by two applications of Fubini's Theorem, $\iint \mu_{k}^{\prime} \circ g d \nu(k) d m(g)=\mu_{X} \times \mu_{Y}$. Thus the mapping $(k, g) \rightarrow \mu_{k}^{\prime} \circ g$ carries $d \nu \times d m$ onto some measure $\sigma^{*}$ on $E$ such that $\int \mu d \sigma^{*}(\mu)$ $=\mu_{X} \times \mu_{Y}$. The measure $\sigma$ is uniquely determined by this property, and so $\sigma=\sigma^{*}$. This same integration property of $\sigma$ shows that the range of the mapping $k \rightarrow \mu_{k}$ must have $\sigma$-measure one. It follows that for almost all $g$, in particular for some $g$, the set $\left\{\mu_{k}^{\prime} \circ g\right\}$ has $\sigma$-measure 1 . The measure $\sigma$ is $G$-invariant and so $\left\{\mu_{k}^{\prime}\right\}$ has $\sigma$ measure 1. This shows that $\mu_{k}=\mu_{k}^{\prime}$ a.e. A routine argument involving properties of standard Borel spaces and measurable sections shows that if $f\left(\mu_{k}\right)=f\left(\mu_{k}^{\prime}\right)$ implies $\mu_{k}=\mu_{k}^{\prime}$ a.e. then there is a null set $N^{*}$ such that if $k \notin N^{*}$ then there is only one $\mu$ with $f(\mu)=k$. Thus $X$ and $Z$ are quasi-disjoint. Q.E.D.

We now discuss preservation of quasi-disjointness under passage to factor processes. As might be expected, this problem is closely connected with the problem of invariant extension of measures. Let $\pi: \Omega_{X} \rightarrow \Omega_{Y}$ be a homomorphism of $X=\left(\Omega_{X}, \mathscr{F}_{X}, T_{X}\right)$ into $Y=\left(\Omega_{Y}, \mathscr{F}_{Y}, T_{Y}\right)$. If $\mu$ is any invariant probability on $X$ then $\mu \circ \pi^{-1}$ is an invariant probability on $Y$. In general, however, $\mu \rightarrow \mu \circ \pi^{-1}$ is not a surjective mapping of the set of invariant measures of $X$ onto those of $Y$, e.g., let $Y$ be trivial and let $X$ be translation on the real line. In fact, we will be concerned with the surjectivity of the mapping $\mu \rightarrow \mu \circ \pi$ when restricted to certain covering measures. Furstenberg [4] dealt with similar difficulties by "topologizing" the processes, and we will do the same. Recall from $\$ 1$ that any process $X$ is isomorphic (mod 0$)$ to a topological process $X^{\prime}$, i.e., $\Omega_{X^{\prime}}$ is a compact metric space, $\mathscr{F}_{X^{\prime}}$ consists of the Borel sets, $T_{X^{\prime}}$ is a homeomorphism, and $\mu_{X^{\prime}}$ is some probability measure. Also recall homomorphisms and eigenfunctions can be taken to be continuous, with no more than a $(\bmod 0)$ error.

Lemma 3.2. Let $X$ and $Y$ be topologized processes. Let $\Omega$ be a compact metric space, and let $T$ be a homeomorphism of $\Omega$ onto itself. Let $\pi_{X}$ and $\pi_{Y}$ be continuous mappings of $\Omega$ onto $\Omega_{X}$ and $\Omega_{Y}$ respectively, such that $T_{X} \pi_{X}=\pi_{X} T$ and $T_{Y} \pi_{Y}=\pi_{Y} T$. Then there exists a T-invariant measure $\mu$ on the Borel field $\mathscr{F}$ of $\Omega$ such that $\mu_{X}=\mu \circ \pi_{X}^{-1}$ and $\mu_{Y}=\mu \circ \pi_{Y}^{-1}$ if and only if there is a point $z$ in $\Omega$ such that $\pi_{X} z$ and $\pi_{Y} z$ are generic points for $X$ and $Y$ respectively. ( $x$ is a generic point for $X$ if

$$
\frac{1}{N} \sum_{k=1}^{N} f\left(T^{k} x\right) \rightarrow \int f d \mu_{X}
$$

for each continuous function $f$ ). 
Proof. If the measure $\mu$ exists then there is some point $z$ in $\Omega$ which is generic for $\mu$, and obviously $\pi_{X} z$ and $\pi_{Y} z$ are generic for $\mu_{X}$ and $\mu_{Y}$. Conversely, suppose $z$ is in $\Omega$ and $\pi_{X} z$ and $\pi_{Y} z$ are generic. Let $F(z)=f_{1}\left(\pi_{X} z\right)+f_{2}\left(\pi_{Y} z\right)$ where $f_{1}$ and $f_{2}$ are continuous functions on $\Omega_{Y}$ and $\Omega_{Z}$. From the generic nature of $\pi_{X} z$ and $\pi_{Y} z$, we conclude

$$
\frac{1}{N} \sum_{k=1}^{N} F\left(T^{k} z\right) \rightarrow \int f_{1} d \mu_{X}+\int f_{2} d \mu_{Y}
$$

Evidently, if $F(z) \equiv 0$ then $\int f_{1} d \mu_{X}+\int f_{2} d \mu_{Y}=0$. It follows easily that

$$
L\left(f_{1} \circ \pi_{X}+f_{2} \circ \pi_{Y}\right)=\int f_{1} d \mu_{X}+\int f_{2} d \mu_{Y}
$$

is a well-defined linear functional on the space of all functions which can be written as $f_{1} \circ \pi_{X}+f_{2} \circ \pi_{Y}$. It is also evident, by observing the Cesaro limit form of the definition, that $L$ is positive, and $L(F \circ T)=L(F)$. If $F$ now is any continuous function, set $p(F)=\inf L\left(F^{\prime}\right)$ where the infimum is taken over the collection of all $F^{\prime}=f_{1} \circ \pi_{X}+f_{2} \circ \pi_{Y}$ such that $F \leqq F^{\prime}$. It is easy to verify $p$ is subadditive, semihomogeneous, and satisfies $p(F \circ T)=p(F)$. It follows from Proposition 5 of Chapter 10 of [13] that $L$ can be extended to the space of all continuous functions on $\Omega$ in such a way that $L(F)=L(F \circ T) \leqq p(F)$. It follows that $L$ is positive, for if $F \geqq 0$ then $-F \leqq 0$ so, by construction of $p, p(-F) \leqq 0$ and so $L(-F) \leqq 0$, i.e., $L(F) \geqq 0$. Since also $L(1)=1$, the functional $L$ is given by some measure $\mu, L(F)$ $=\int F d \mu$. It is easy to verify $\mu$ has the required properties. Q.E.D.

Proposition 3.3. Let $X, Y$, and $Z$ be topologized processes, and let $\pi_{X}: X \rightarrow Y$ be a continuous homomorphism. Let $\mu$ be an invariant covering measure on $Y \times Z$. Then there is an invariant covering measure $\mu_{1}$ on $X \times Z$ such that $\mu_{1} \circ \pi^{-1}=\mu$ $\left(\pi=\pi_{X} \times\right.$ id).

Proof. We let $\Omega=\{(x, y, z) \mid \pi x=y \quad$ (and $\quad(x, y, z) \in X \times Y \times Z)\}$. We let $\Delta=\{(y, z) \mid(y, z)$ is generic for $N\}$. Then $\Delta$ has $\mu$-measure 1 , and it follows easily that the image of $\Delta$ in $Y$, under the natural projection, has $\mu_{Y}$ measure one (precisely, the image is a $\mu$-measurable set of measure one). In a similar manner, the image under $\pi_{X}$ of the set of generic points for $\mu_{X}$ has measure one. It follows that there is a point $\left(x_{0}, y_{0}, z_{0}\right)$ in $\Omega$ such that $x_{0}$ is generic for $\mu_{X}$ and $\left(y_{0}, z_{0}\right)$ is generic for $\mu$. From the preceding lemma, a $T$-invariant measure $\mu^{\prime}$ exists on $\Omega$ which projects onto $\mu_{X}$ and $\mu$ under the natural mappings. We set $\mu_{1}=\mu^{\prime} \circ \pi^{\prime}$ where $\pi^{\prime}(x, y, z)=(x, z)$. Evidently, $\mu_{1} \circ \pi^{-1}=\mu_{X}$, and the fact that $\mu_{1}$ projects onto $\mu_{Z}$ follows from the facts that $\mu$ does and that the following diagram commutes:

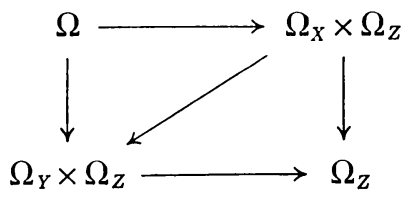


Corollary 3.4. Let $X, Y, Z$ and $\pi$ be as in the preceding proposition and let $\mu$ be an ergodic invariant covering measure on $Y \times Z$. Then there is an ergodic invariant covering measure $\mu_{1}$ on $X \times Z$ such that $\mu_{1} \circ \pi^{-1}=\mu\left(\pi=\pi_{X} \times \mathrm{id}\right)$.

Proof. Given $\mu$, let $V$ consist of all invariant covering measures on $X \times Z$ which project, under $\pi$, into $Y$. By the preceding proposition, $V$ is nonempty. Evidently, $V$ is convex and it is compact in the weak* topology. By the Krein-Milman Theorem there is an extreme point $\mu_{1}$ in $V$. What we must now show is that $\mu_{1}$ is extreme in the collection of all invariant measures. Suppose $\mu_{1}=t \nu+(1-t) \nu^{\prime}$, $0<t<1$, and $\nu$ and $\nu^{\prime}$ are invariant measures on $X \times Z$. Let $\pi_{1}(x, z)=x$. We see $\mu_{X}=\mu_{1} \circ \pi_{1}^{-1}=t\left(\nu \circ \pi_{1}^{-1}\right)+(1-t)\left(\nu^{\prime} \circ \pi_{1}^{-1}\right)$. Now $\nu \circ \pi_{1}^{-1}$ and $\nu^{\prime} \circ \pi_{1}^{-1}$ are $T_{X}$ invariant measures and $\mu_{X}$ is ergodic so, since $0<t<1, \nu \circ \pi_{1}^{-1}$ and $\nu^{\prime} \circ \pi_{1}^{-1}$ are both equal to $\mu_{X}$, i.e., $\nu$ and $\nu^{\prime}$ cover $\mu_{X}$. Similarly, $\nu$ and $\nu^{\prime}$ cover $\mu_{Y}$ and also projects onto $\mu$. This shows $\nu$ and $\nu^{\prime}$ are in $V$, and so $\nu=\nu^{\prime}$. Thus $\mu_{1}$ is extreme in the collection of all invariant measures, i.e., ergodic. Q.E.D.

THEOREM 3.5. Let $\pi_{X}: X \rightarrow Y$ be a homomorphism of the ergodic process $X$ into $Y$ and let $Z$ be an ergodic process which is quasi-disjoint from $X$. Then $Z$ is quasidisjoint from $Y$.

Proof. We can assume $X, Y$, and $Z$ have been topologized (compact metric spaces with homeomorphisms), that each eigenvalue possesses a continuous eigenfunction, that $\pi$ is continuous and surjective, and that the homomorphisms $\alpha: X \rightarrow K(X, Z), \alpha^{\prime}: Y \rightarrow K(Y, Z), \beta: Z \rightarrow K(X, Z)$ and $\beta^{\prime}: Z \rightarrow K(Y, Z)$ are continuous and surjective. We will first prove this theorem under the additional assumption that $K(X, Z)=K(Y, Z)$ i.e., $\mathscr{P}(X) \cap \mathscr{P}(Z)=\mathscr{P}(Y) \cap \mathscr{P}(Z)$.

We set, as usual, $\gamma(x, z)=\alpha(x)-\beta(z)$ and $\gamma^{\prime}(y, z)=\alpha^{\prime}(y)-\beta^{\prime}(z)$. We can, and do, suppose $\alpha, \alpha^{\prime}, \beta$ and $\beta^{\prime}$ have been chosen so that $\alpha^{\prime} \circ \pi_{X}=\alpha$ and $\beta^{\prime}=\beta$. It is easy now to verify that $\gamma^{-1}(k)=\pi^{-1} \gamma^{\prime-1}(k)$ (where $\pi=\pi_{X} \times$ id). If $\mu$ is any invariant ergodic cover (of $\mu_{Y}$ and $\mu_{Z}$ ) on $Y \times Z$, then, by the preceding corollary, $\mu=\mu_{1} \circ \pi^{-1}$ for some ergodic cover (of $\mu_{X}$ and $\mu_{Z}$ ) on $X \times Z$. Moreover, if $\mu$ is supported by $\gamma^{\prime-1}(k)$, then $\mu_{1}$ will be supported by $\pi^{-1} \gamma^{-1}(k)=\gamma^{-1}(k)$. Evidently, the measures $\nu=\left(\mu_{X} \times \mu_{Z}\right) \circ \gamma^{-1}$ and $\nu^{\prime}=\left(\mu_{Y} \times \mu_{Z}\right) \circ \gamma^{\prime-1}$ are the same measure, so that if for $\nu$-almost every $k$ the set $\gamma^{-1}(k)$ supports only one invariant cover $\mu_{1}$, then for $\nu^{\prime}$-almost every $k$ the set $\gamma^{\prime-1}(k)$ supports only one invariant cover $\mu$.

Now drop the assumption that $K(X, Z)=K(Y, Z)$. We let $\pi_{K}$ be the natural mapping of $K(X, Z)$ onto $K(Y, Z)\left(K=\right.$ dual $\Lambda$, and $\pi_{K}(k)$ is $k$, restricted to $\mathscr{P}(Y) \cap \mathscr{P}(Z)$ ). We can assume (see the discussion in Theorem 3.1) that $\alpha^{\prime} \circ \pi_{X}$ $=\pi_{K} \circ \alpha, \beta^{\prime}=\pi_{K} \circ \beta$. Let $K=K(X, Z)$ and construct a new process $X$ as follows: Obviously $K(Y, Z)$ is the greatest common Kronecker factor of $Y$ and $K$, and $\alpha^{\prime}: Y \rightarrow K(Y, Z), \pi_{K}: K \rightarrow K(Y, Z)$ are homomorphisms. Since a Kronecker process is a group extension of the trivial process, we know $Y$ and $K$ are quasidisjoint. Thus, for some $k_{0}$ in $K(Y, Z)$, the set

$$
\Omega=\left\{(y, k) \in \Omega_{Y} \times \Omega_{K} \mid \alpha^{\prime}(y)=\pi_{K} k+k_{0}\right\}
$$


supports exactly one $T_{Y} \times T_{K}$ invariant measure $\mu$ which covers both $\mu_{Y}$ and $\mu_{K}$ (recall here $T_{K}$ is a rotation on the group $\Omega_{K}$, and $\mu_{K}$ is Haar measure). We set $X_{1}=\left(\Omega, \mathscr{F}, T_{Y} \times T_{K}, \mu\right)$ where $\mathscr{F}$, of course, consists of the Borel $\sigma$-field. The mapping $x \rightarrow\left(\pi_{X}, \alpha(x)-k_{0}^{\prime}\right)$, where $\pi_{K}\left(k_{0}^{\prime}\right)=k_{0}$, is evidently a homomorphism of $X$ into $X_{1}$ (the commutativity of the mappings is obvious, and the image of $\mu_{X}$ on $X_{1}$ clearly covers $\mu_{X}$ and the image on $K$ must cover $\mu_{K}$ since $K$ is uniquely ergodic. Now recall there is only one measure, $\mu$, on $X_{1}$ with these properties). Also, since $K$ is a factor of $X_{1}, \mathscr{P}\left(X_{1}\right) \supseteq \mathscr{P}(K)=\mathscr{P}(X) \cap \mathscr{P}(Z)$ so $\mathscr{P}\left(X_{1}\right) \cap \mathscr{P}(Z) \supseteq \mathscr{P}(X)$ $\cap \mathscr{P}(Z)$. The reverse inequality follows from the fact that $X_{1}$ is a factor of $X$, and so $K\left(X_{1}, Z\right)=K(X, Z)$. We have discussed this special case, and so we know $X_{1}$ is quasi-disjoint from $Z$.

Now let $N$ be the kernel of the mapping $\pi_{K}$. If $n \in N$ then $\pi_{K}(k+n)=\pi_{K}(k)$ and so $(y, k) \rightarrow(y, k+n)$ is a bijection of $\Omega$ onto itself. Moreover, the measure $\mu$ on $\Omega$ is carried by this mapping into a $T$-invariant cover, and by the uniqueness property of $\mu$, we conclude $(y, k) \rightarrow(y, k+n)$ is $\mu$-measure preserving. Also, since $T_{K}(k+n)$ $=T_{K}(k)+n$, the mapping being studied commutes with $T_{Y} \times T_{K}$. Let $\pi_{1}: X_{1} \rightarrow Y$ be $\pi_{1}(y, k)=y$ and let $\alpha_{1}: X_{1} \rightarrow K$ be $\alpha_{1}(y, k)=k+k_{0}^{\prime}$ (here, as before, $\pi_{K}\left(k_{0}^{\prime}\right)=k_{0}$ and $k_{0}$ is the point appearing in the definition of $\left.\Omega\right)$. We already know what is meant by $\beta: Z \rightarrow K, \beta^{\prime}: Z \rightarrow K(Y, Z), \alpha^{\prime}: Y \rightarrow K(Y, Z)$ and $\gamma^{\prime}(y, z)=\alpha^{\prime}(y)-\beta^{\prime}(z)$. Let $\gamma_{1}(y, k, z)=\alpha_{1}(y, k)-\beta(z)$. Observe that $\gamma^{\prime} \circ\left(\pi_{1} \times\right.$ id $)(y, k, z)=\alpha^{\prime}(y)-\beta^{\prime}(z)$ while $\left(\pi_{K} \circ \gamma\right)(y, k, z)=k+k_{0}^{\prime}-\beta(z)$, restricted to $\mathscr{P}(Y) \cap \mathscr{P}(Z)$. It follows from the definition of $\Omega$ (and of $\left.k_{0}^{\prime}\right)$ that $\gamma^{\prime} \circ\left(\pi_{1} \times\right.$ id) $=\pi_{K} \circ \gamma$. What we need to show is that for $\nu^{\prime}=\mu_{Y} \times \mu_{Z} \circ \gamma^{\prime-1}$-almost every $k$ in $K(Y, Z),\left(\gamma^{\prime}\right)^{-1}(k)$ supports only one ergodic invariant covering measure. Any such ergodic invariant covering measure on $Y \times Z$ can be lifted to an ergodic invariant covering measure on $X_{1} \times Z$. The ergodicity implies that the support will be on some $\gamma_{1}^{-1}\left(k^{\prime}\right), k^{\prime} \in K$, and it is not hard to see that $\pi_{K}\left(k^{\prime}\right)=k$. Now for almost every $k$ there is some $k^{\prime \prime}$ such that $\pi_{K}\left(k^{\prime \prime}\right)=k$ and $\gamma_{1}^{-1}\left(k^{\prime \prime}\right)$ supports only one ergodic invariant covering measure. Now suppose $\pi_{K}\left(k^{\prime}\right)=\pi_{K}\left(k^{\prime \prime}\right)=k, \mu$ is an ergodic invariant covering measure supported by $\gamma^{\prime-1}(k), \mu_{1}$ is an ergodic invariant covering measure supported by $\gamma^{-1}\left(k^{\prime}\right)$ which projects onto $\mu_{1}$, i.e., $\mu_{1} \circ\left(\pi_{1} \times \text { id }\right)^{-1}=\mu$, and $\gamma^{-1}\left(k^{\prime \prime}\right)$ supports only one ergodic invariant covering measure. Let $n=k^{\prime \prime}-k^{\prime}$ and observe $n \in N$. Let $\eta((y, k, z))=(y, k+n, z)$ and observe $\mu_{1} \circ \eta^{-1}$ is an ergodic invariant covering measure supported on $\gamma^{-1}\left(k^{\prime \prime}\right)$ and that $\mu_{1} \circ \eta^{-1} \circ\left(\pi_{1} \times \mathrm{id}\right)^{-1}=\mu_{1} \circ\left(\left(\pi_{1} \times \mathrm{id}\right) \circ \eta\right)^{-1}$ $=\mu$. Thus any ergodic invariant covering measure on $\gamma^{\prime-1}(k)$ is the image of the unique such measure on $\gamma^{-1}\left(k^{\prime \prime}\right)$, which establishes the uniqueness of $\mu$. Q.E.D.

\section{REFERENCES}

1. S. K. Berberian, Notes on spectral theory, Van Nostrand Math. Studies, no. 5, Van Nostrand, Princeton, N. J., 1966. MR 32 \#8170.

2. J. R. Blum and D. L. Hanson, On invariant probability measures. I, Pacific J. Math. 10 (1960), 1125-1129. MR 24 \#A3260. 
3. N. Bourbaki, Les structures fondamentales de l'analyse. Livre VI: Intégration, Actualités Sci. Indust., no. 1175, Hermann, Paris, 1952. MR 14, 960.

4. H. Furstenberg, Disjointness in ergodic theory, minimal sets, and a problem in Diophantine approximation, Math. Systems Theory 1 (1967), 1-49. MR 35 \#4369.

5. F. J. Hahn, On affine transformations of compact abelian groups, Amer. J. Math. 85 (1963), 428-446. MR 27 \#5889.

6. - Minimal dynamical systems with quasi-discrete spectrum, J. London Math. Soc. 40 (1965), 309-323. MR 30 \#5292.

7. - Some characteristic properties of dynamical systems with quasi-discrete spectra, Math. Systems Theory 2 (1968), 179-190. MR 37 \#6435.

8. P. R. Halmos, Lectures on ergodic theory, Publ. Math. Soc. Japan, no. 3, Math. Soc. Japan, Tokyo, 1956; reprint, Chelsea, New York, 1960. MR 20 \#3958.

9. - Introduction to Hilbert space and the theory of spectral multiplicity, Chelsea, New York, 1951. MR 13, 563.

10. '-, Measure theory, Van Nostrand, Princeton, N. J., 1950. MR 11, 504.

11. G. W. Mackey, Borel structure in groups and their duals, Trans. Amer. Math. Soc. 85 (1957), 134-165. MR 19, 752.

12. P. A. Meyer, Probability and potentials, Blaisdell, Waltham, Mass., 1966. MR 34 \#5119.

13. H. L. Royden, Real analysis, 2nd ed., Macmillan, New York, 1968.

14. V. S. Varadarajan, Groups of automorphisms of Borel spaces, Trans. Amer. Math. Soc. 109 (1963), 191-220. MR 28 \#3139.

University of Maryland, College Park, Maryland 20842 